\title{
Remote sensing of evapotranspiration and carbon uptake at Harvard Forest
}

\author{
Qilong Min* and Bing Lin+ \\ *Atmospheric Sciences Research Center, State University of New York, Albany \\ + Radiation and Aerosols Branch, NASA Langley Research Center, Hampton, Virginia
}

\begin{abstract}
:
A land surface vegetation index, defined as the difference of microwave land surface emissivity at 19 and 37 $\mathrm{GHz}$, was calculated for a heavily forested area in north central Massachusetts. The microwave emissivity difference vegetation index (EDVI) was estimated from satellite SSM/I measurements at the defined wavelengths and used to estimate land surface turbulent fluxes. Narrowband visible and infrared measurements and broadband solar radiation observations were used in the EDVI retrievals and turbulent flux estimations. The EDVI values represent physical properties of crown vegetation such as vegetation water content of crown canopies. The collocated land surface turbulent and radiative fluxes were empirically linked together by the EDVI values. The EDVI values are statistically sensitive to evapotranspiration fractions $(\mathrm{EF})$ with a correlation coefficient $(R)$ greater than 0.79 under all-sky conditions. For clear skies, EDVI estimates exhibit a stronger relationship with EF than normalized difference vegetation index (NDVI). Furthermore, the products of EDVI and input energy (solar and photosynthetically-active radiation) are statistically significantly correlated to evapotranspiration $(R=0.95)$ and $\mathrm{CO}_{2}$ uptake flux $(R=0.74)$, respectively.
\end{abstract}




\section{Introduction}

Forests modulate surface and boundary layer energy and hydrological budgets on local, regional, and continental scales, primarily through evapotranspiration (ET) processes. Characterization of ET processes is essential in understanding the energy and water cycle of the responses of climate and terrestrial ecological systems to climate change and variation. However, there is a lack of accurate large-scale measurements of ecosystem exchange processes. Satellite remote sensing cannot measure surface turbulent flux exchanges directly. Researches are focused on parameterizations of the exchange processes, such as canopy resistance, that may be remotely sensed by satellites [Nishida et al. 2003]. Most of existing satellite remote sensing techniques for ET estimations are based on measurements at visible and near-infrared wavelengths, such as the normalized difference vegetation index (NDVI), as the spectral measurements are highly correlated to the absorbed fraction of photosyntheticallyactive radiation (PAR) [Asrar et al. 1984; Sellers 1985; Myneni et al. 1995; Granger 2000; Jiang and Islam, 2003; Nishida et al, 2003]. Since these measurements are strongly influenced by clouds and aerosols, their capability of monitoring ET under all-weather conditions and accurately estimating the spring onset and growing season duration is limited.

At microwave wavelengths, vegetation plays a significant role in surface microwave emission. Physical properties of vegetation, such as, plant water content, vegetation areal coverage and vertical structure, vegetation phenology, and physical temperature, are major factors in determining satellite measured radiances [Wigneron et al., 1993; Njoku, 1999; Wigneron et al., 2003 and reference therein]. More importantly, microwave land surface emissivity (MLSE) can be derived from satellite measurements during both day and night times under nonprecipitating conditions. This study evaluates land surface properties and atmosphere-ecosystem exchanges using microwave measurements. MLSE values are retrieved from a combination of visible, infrared, and microwave measurements from surface and satellites. The visible and infrared remote sensing data are mainly used for obtaining cloud properties that are important for microwave atmospheric absorption corrections in MLSE estimations. We found that MLSE values can serve as an empirical proportional factor between land surface turbulent and radiative fluxes over the dense forest area, Harvard Forest. 


\section{Methodology and measurements}

\subsection{Microwave emissivity difference vegetation index (EDVI)}

There is a semi-empirical relation between the optical depth at microwave wavelengths and vegetation water content (VWC), which varies systematically with both wavelength and canopy structure [Jackson and Schmugge, 1991]. The microwave surface emission above a canopy is an integration of the microwave radiation from the whole canopy vertical profile weighted by its transmission. The emissivity observed at longer wavelengths with a weaker attenuation by the canopy generally represents an effectively thicker layer than those observed at shorter and stronger attenuation wavelengths. Some studies even found that the brightness temperature difference at two microwave wavelengths has certain capability to classify land surface type and to determine forest characteristics [Neale et al. 1990; Pulliainen et al. 1999; Macelloni et al. 2003]. Thus, a new parameter based on the microwave land surface emissivity difference between two wavelengths is introduced to indicate VWC and other vegetation properties of the canopy with a minimal influence of the soil emission underneath vegetation canopy. Analogous to NDVI and the frequency index of Macelloni et al., the new parameter, microwave emissivity difference vegetation index (EDVI), is defined as:

$$
E D V I_{P}=\frac{M L S E_{P}^{A}-M L S E_{P}^{B}}{0.5\left(M L S E_{P}^{A}+M L S E_{P}^{B}\right)} .
$$

where $p$ represents a polarization at vertical or horizontal direction, and A and B indicate the two wavelengths of microwave measurements. EDVI data derived from the 19.4 and $37.0 \mathrm{GHz}$ Special Sensor Microwave/Imager (SSM/I) measurements of Defense Meteorological Satellite Program (DMSP) satellites are analyzed to investigate their potential for detecting vegetation physiology changes and estimating land-atmosphere exchange. $M L S E_{P}^{19}$ represents the thicker effective emission layer deeper into the canopy while $M L S E_{P}^{37}$ represents the thinner one, due to higher attenuation effects at higher frequencies. Thus, EDVI represents the canopy properties of VWC and structure of two effective emission layers.

When a vegetated surface layer is very thick (e.g., a forest), microwave emissivity from the vegetated layer depends largely on properties of the canopy [Jackson et al, 1984, Jackson and Schmugge, 1991, Paloscia, 1995; 
Wigneron et al. 2003]. Radiation at the frequencies of SSM/I scantily penetrates through the forest. The microwave emission at high frequencies above from forest canopies is more profoundly affected by the upper potion of canopies (crown layers) than the trunks and stems. Therefore, we subdivide the forest canopy into two main regimes: crown and trunk (Figure 1) [Ferrazzoli and Guerriero, 1996]. Thus, the basic model for forest microwave radiative transfer consists three layers: soil + trunk + leave, shown in the left side of Figure 1. Furthermore, since the physical sizes of trunks and primary branches are much larger than the wavelengths of SSM/I, the scattering effects of the trunk layer are substantially weaker than that of leaves, needles, and twigs in the crown. The size parameter differences of trunk and crown layer make the radiative processes in these layers reach geometry optics limits and stay in Mie scattering regime, respectively, resulting different single scattering albedos for trunk and crown layers. For a strong absorption medium like vegetation in the microwave wavelengths, the radiative process approaches geometry optics limits when the size parameter is greater than $\sim 10$, i.e., the size of diameter of trunk and primary branch is larger than $\sim 5 \mathrm{~cm}$ in the $\mathrm{SSM} / \mathrm{I}$ wavelengths [Hanson and Travis, 1974]. Since the scattering effects, especially the detailed multiple scattering effect caused by diffraction and interference in physical optics, can be approximated through simple geometry optics or be neglected, the trunk layer can be dealt as an absorption/emission layer with a lot of holes where the microwave radiation from soil can directly pass through, i.e., we can use emissivity values of trunks in the trunk occupied areas to describe the microwave radiation above the trunks. However, the crown layer is significantly different from the trunk layer. The leave and small branch scatter microwave radiation considerably. The multiple scattering by the crown has to be accounted for. In the sense of combined soil and trunk layers, the microwave radiation can be considered as the sum of microwave radiation of soil and trunk weighted by the areal coverage of trunks and open soil areas. At Harvard Forest, both total and crown layer vegetation areal coverage values are very high (about $100 \%$ ) during grown seasons. Although the soil-trunk layer is heterogeneous, for trunk (and primary branches) layer itself, the fractional areal coverage is estimated over $80 \%$ based on observed albedo values under snowcover conditions [Robinson et al, 1993]. Thus, we simply treat the trunk layer as an absorption and emission layer and integrate it with the soil layer as a single conceptual soil-trunk layer for microwave radiative transfer simulations, as illustrated in the right side of Fig. 1. Therefore, to understand the impact of soil-forest interaction on the microwave land surface emissivity and EDVI, we use a simple two-layer model that considers the crown 
layer of the forest to be a homogenous absorbing and scattering medium above an emission layer of the soil-trunk. The microwave emissivity of a forest-covered land surface is expressed as [Paloscia, 1995]

$\varepsilon_{c}=1-\left(1-\varepsilon_{s}\right) \exp (-2 \tau / \mu)-\omega(1-\exp (-\tau / \mu))\left[1+\left(1-\varepsilon_{s}\right) \exp (-\tau / \mu)\right]$

where $\tau$ is the optical depth of the crown layer; $\omega$ is the averaged single scattering albedo of the materials such as leafs and small branches within the crown layer; $\varepsilon_{\mathrm{s}}$ is the emissivity of the conceptual soil-trunk layer and a function of soil moisture, the fractional coverage of trunks, trunk water content and structure; $\mu$ is radiation direction.

In our following simulation, the optical depth of the crown is linearly correlated to vegetation water content (VWC) or crown water content as $\tau=0.1 *(f / 1.4) * \mathrm{VWC}$ (f is the frequency) [Choudhury, 1995]. The single scattering albedo at 19.4 and $37 \mathrm{GHz}$ are assumed to be 0.07 and 0.09 , respectively [Paloscia, 1995]. The soiltrunk emissivities at 19.4 and $37 \mathrm{Ghz}$ are assumed to be 0.960 and 0.958 , the mean SSM/I retrieved values at both wavelengths before the spring onset at Harvard Forest. The retrieval algorithm of MLSE is described in the next section. Before the emergence of leaves (initial bud break), the retrieved emissivities are truly integrated emissivities of trunk layer with underneath soil layer. This approach of trunk-soil layer emissivities actually assumes that the VWC of crown layer is zero, although small branches and twigs contain some water. Although precipitation and evaporation may alter the soil-trunk emissivity (mainly soil emissivity), the trunk layer generally effectively damps the large change of soil emissivity caused by variations in soil moisture because of over $80 \%$ coverage of trunks and primary branches over the forest area. For the trunk itself, precipitation has much less influence on the trunk emissivity due to quick evaporation of trunk skin water. Based on the dynamic range of the retrieved emissivity, we also simulate the cases with \pm 0.15 changes in the soil-trunk emissivity. The $53^{\circ}$ view angle of SSM/I is used in the simulation. Figure 2 shows simulated MLSE and newly defined EDVI as a function of VWC for various soil-trunk emissivities. For wet soil-trunk cases with soil-trunk emissivites below 0.96, when VWC is relatively small the emission increases with VWC due to the scattering and emission effects of crown, and then decreases because of strong absorption of crown to soil-trunk emission as VWC increases (left panel). For dry soil-trunk case with the soil-trunk emissivity of 0.975 , the emissivity of a canopy-covered land surface decreases monotonically with VWC. This result illustrates that MLSE at both wavelengths are sensitive to the 
soil-trunk emissivity, and are nonlinearly related to VWC. With single emissivity value, there are even two possible VWC solutions from the simulation. The EDVI (right panel), however, is insensitive to the soil-trunk emissivity and is linearly related to VWC for small VWC values. The upper and lower curves provide the uncertainty in the estimation associated with possible changes of soil-trunk moisture. For large VWC, the crown fully block the emission of the soil-trunk layer, so that the emissivity from crown would mainly depend on the properties of the crown and will converge to a value, 1- $\omega$, independent of soil-trunk moisture. In the large VWC conditions, EDVI is also saturated and becomes the difference of the single scattering albedos at the two wavelengths, representing dielectric constants and vegetation structures of crown [Van de Griend and Wigneron, 2004]. The dielectric constant is directly related to moisture content of vegetation and the vegetation structures (leaf area and thickness) has strongly influences on crown conductance [Ulaby et al, 1986]. For VWC of crown layers, some observations found that VWC values were small and within the range of $0.5 \mathrm{~kg} / \mathrm{m}^{2}$ [Zarco-Tejada et al., 2003; Sims and Gamon, 2003]. Others [Jackson et al, 2004] suggested that the values could be much larger than $0.5 \mathrm{~kg} / \mathrm{m}^{2}$. Our model simulations were made in small VWC dynamic ranges. Although the model EDVI

results saturate at about the value of $0.5 \mathrm{~kg} / \mathrm{m}^{2}$, it may not reflect real world since the model does not account for multiple scattering effects and only has the $1^{\text {st }}$ order scattering influences (single scattering). With considerations of full multiple scattering, the increase of EDVI with VWC is going to be slower than what we simulated (Fig.2b), and the saturation point of EDVI on VWC should be much higher. Nonetheless, this model illustrates the fundamental physics of linkage of EDVI to the crown VWC. For vegetation types other than forests, the entire vegetation canopy can be treated as one single layer because the sizes of the stalks are small or comparable to the wavelengths of SSM/I. Therefore, the index of EDVI developed here can directly apply to some vegetation types, such as grasses.

\subsection{MLSE and Harvard Forest}

The Harvard Forest Environmental Monitoring Station (EMS) is located in north-central Massachusetts (42.54N, $78.18 \mathrm{~W}$ ). The forest is $50-70$ years old and contains a mixture of red oak, red maple, and hemlock with an average tree height of $24 \mathrm{~m}$. The site has been equipped with a suite of radiation and turbulent flux measurements since 1991 [Wofsy et al., 1993; Moore et al. 1996]. The long-term turbulent exchange, such as latent heat and 
$\mathrm{CO}_{2}$ fluxes, and radiation and ground heat flux measurements sampled every 30 min at EMS provide us the opportunity to investigate processes of evapotranspiration, $\mathrm{CO}_{2}$ uptake, and photosynthesis [Min, 2005].

MLSE values were retrieved from the SSM/I data of DMSP F13 and F14 satellites from 1999 to 2001 at the Harvard Forest site for all SSM/I wavelengths and polarizations using the technique developed by Lin and Minnis [2000]. The SSM/I data were spatially $(20 \mathrm{~km})$ and temporally $(15 \mathrm{~min})$ collocated with surface measurements at the Harvard Forest EMS for the retrievals of MLSE. These MLSE values are estimated based on an atmospheric microwave radiative transfer (MWRT) model [Lin et al., 1998], which accurately accounts for the atmospheric absorption and emission of gases and clouds, especially the temperature and pressure dependences of these radiative properties [Lin et al., 2001]. Only non-precipitating cases were analyzed to avoid the complexity of microwave scattering and the dependence of observed radiances on precipitating hydrometeors. The major inputs of the model are effective land surface skin temperature, column water vapor (CWV), cloud water amount, surface air temperature and pressure. The European Center for Medium-range Weather Forecasts (ECMWF) assimilation data is used to estimate CWV values. Atmospheric optical depths inferred from total shortwave measurements assuming $8 \mu \mathrm{m}$ cloud effective radius were converted to cloud water amount. The vertical distributions of atmospheric temperature, pressure and gas abundance were constructed based on climatological profiles [McClatchy et al. 1972] and interpolated to conform to the surface measurements of temperature and pressure and ECMWF CWV values. Since the coverage of forest at Harvard Forest within SSM/I footprints is fairly homogeneous, the possible impact of forest heterogeneity on the emissivity is minimal (only some small roads, lakes, and villages are within the region).

\section{Results}

During transition periods of early growing season and leaf senescence, transpiration plays a dominant role in regulating ET processes. Since leaves and needles in the crown of forests are major players in photosynthesis, the EDVI represents key physical properties associated with vegetation water content of the canopy in atmosphere and land surface exchange processes. Figure 3 shows retrieved vertically polarized EDVI at the Harvard Forest site during the growing season of 1999. In the plot, we also include NDVI values derived from Advanced Very 
High Resolution Radiometer (AVHRR; http://daac.gsfc.nasa.gov/data/dataset/avhrr/). The satellite retrieved EDVI varies from a very small number to $\sim 0.025$, which is within our simulated EDVI range (Fig. 2 ). The vertical dotted line indicates the averaged day of year (DOY) of observed initial bud break over four representative species at Harvard Forest (red oak, white oak, red maple, and yellow birch) [O'keefe, 2004]. After the spring onset DOY 129, $\mathrm{EDVI}_{\mathrm{V}}$ increased sharply in the first 20 days due to rapid increase of moisture content of vegetation caused by leaf emergence. It took an additional month for $\mathrm{EDVI}_{\mathrm{V}}$ to reach its maximum. Although there are no in-situ measurements of VWC values of the forest, the general trend and magnitude of $\mathrm{EDVI}_{\mathrm{V}}$ values consistent with the prediction of the simple two-layer model when VWC is small. During the process of senescence, $\mathrm{EDVI}_{\mathrm{V}}$ decreased back to minimum values. The satellite estimated $\mathrm{EDVI}_{\mathrm{V}}$ captures the seasonal changes of leaf physiology, the overriding feature of deciduous forest canopy. The NDVI values derived from AVHRR at optical wavelengths also link to the characteristics of canopy properties, as a result of absorption and reflection of canopy. However, it is substantially influenced by the atmospheric conditions and can only be derived on clear-sky conditions. Furthermore, after onset of leaf senescence at the end of growing season, the NDVI values was reduced but still somewhat high due to the physical presence of some leaves.

The ET processes depend upon the availability of both water within the soil and canopy and energy to change the water state along with forest-atmosphere dynamics. The net energy exchange is a function of environmental factors such as incoming radiation, which shows large diurnal changes responding to variations in the solar zenith angle and cloud coverage. To understand the linkage of ET fluxes to surface properties, some studies have used the evaporation fraction (EF) as an index for ET [Shuttleworth et al. 1989, Jiang and Islam, 2003, Nishida et al. 2003]:

$E F=E T /\left(R_{N}-G\right)$

where $R_{N}$ and $G$ are the net radiation and the ground heat flux, respectively. Since EF is nearly constant during daylight hours [Shuttleworth et al., 1989; Crago, 1996], it can be used for scaling instantaneous satellite observations to longer time periods (daily or daytime). Moreover, EF is directly related to the surface energy partition or Bowen Ratio (BR) by $E F=1 /(1+B R)$. We use the ET fluxes and total available energy $\left(R_{N}-G\right)$ 
simultaneously measured between 10 and 15 local times (LT) at the site tower and Eq. 3 to estimate the EF values at Harvard Forest.

Comparing the satellite EDVI retrievals with the derived EF values, it indicates that there is a strong (albeit nonlinear) relationship between the two. The correlation coefficient $(R)$ between the EF and EDVI is 0.79 for vertical polarization and 0.72 for horizontal polarization (Figs. $4 \mathrm{a}$ and $4 \mathrm{~b}$ ). The horizontal component of the EDVI is generally more sensitive to a broader range of canopy properties, such as VWC, canopy leaf/stem structure, and orientation, with a larger dynamic range. The crosstalk among these canopy properties may reduce the correlation of the horizontally polarized EDVI with the specific variable EF, i.e, evapotranspiration. We have also tested other possible indexes, such as the frequency polarization index FPI $=\left[\left(\operatorname{Tb}^{19}{ }_{\mathrm{v}}-\mathrm{Tb}^{37}{ }_{\mathrm{v}}\right)+\left(\mathrm{Tb}^{19}{ }_{\mathrm{v}}-\mathrm{Tb}^{19}{ }_{\mathrm{h}}\right)\right] / 2$ for snow parameters [Pulliainen et al. 1999; Macelloni et al. 2003], and found that the EDVI $\mathrm{V}$ has the greatest correlation coefficients. Thus, we will mainly use the $\mathrm{EDVI}_{\mathrm{V}}$ in the following analysis.

During the growing season when the canopy resistance was low, changes in other environmental parameters (such as water vapor deficit, near surface wind speed and aerodynamic conductance) have impacts on the ET process (i.e. $\mathrm{EF}$ increased sharply with $\mathrm{EDVI}_{\mathrm{V}}$ with great variability). The solid dots in Figure 4 indicate the cases under clear-sky conditions when NDVI measurements were available. As shown in Figures 5a and 5b, the correlation $(\mathrm{R}=0.52)$ of EF with NDVI is not as strong as that with $\mathrm{EDVI}_{\mathrm{V}}$ under clear-sky conditions $(\mathrm{R}=0.86)$ and even weaker than that with $\mathrm{EDVI}_{\mathrm{V}}$ under all-weather conditions $(\mathrm{R}=0.79)$, which may suggest that $\mathrm{EDVI}_{\mathrm{V}}$ is more sensitive to EF, or moisture content of vegetation in the crown canopy, than NDVI. Note that EDVI $\mathrm{V}_{\mathrm{V}}$ and NDVI values are significantly correlated with a correlation coefficient of 0.83 .

Quantifications of carbon stocks and fluxes are a key objective for ecosystem and carbon cycle studies. The ratio of $\mathrm{CO}_{2}$ uptake fluxes to PAR depends on both vegetation and aerodynamic characteristics [Waring and Running, 1998]. Since NDVI is chlorophyll sensitive, the correlation of NDVI with the uptake ratio ( $\mathrm{R}=0.85$; Figure $6 \mathrm{a})$ is expected to be better than that with $\mathrm{EF}\left(\mathrm{R}=0.52\right.$; Fig. 5a). Even though, $\mathrm{EDVI}_{\mathrm{V}}$ shows a better relationship with the uptake ratio ( $\mathrm{R}=0.92$; Figure $6 \mathrm{~b}$ ). One possible reason for this is that the penetration and reflection of photons at the optical wavelengths are limited to the top portion of crown layer of the forest, resulting in NDVI's less 
sensitivity to leaf development of the entire canopy than $\mathrm{EDVI}_{\mathrm{V}}$ when leaf area coverage is high.

The availability of energy to the canopy, i.e., $R_{N}-G$, is also an important parameter for ET estimations. An accurate estimation of $R_{N}-G$ is not easy via satellite remote sensing. However, the primary surface energy source is surface shortwave (SW) radiation, which can be inferred from satellite measurements with reasonable accuracy [Pinker et al, 2003]. Following Jiang and Islam [2003], we only use the surface measured SW radiation and $\mathrm{EDVI}_{\mathrm{V}}$ to estimate ET, neglecting the ground heat flux in calculations of total available energy. This approach provides an opportunity to estimate ET fluxes using only satellite data. As discussed previously, the $\mathrm{EDVI}_{\mathrm{V}}$ has a nonlinear relationship with the EF. To demonstrate the basic relationship, we use a simplest linear assumption and define an ET index as $\mathrm{ETI}=\mathrm{EDVI}_{\mathrm{V}} * \mathrm{SW}$. Figure 7a shows a scattergram of observed ET with ETI. There is a statistically significant correlation between $\mathrm{EDVI}_{\mathrm{V}}$ and ET values ( $R$ is about 0.95$)$. ET fluxes are essentially decided by SW energy inputs and vegetation states. During current ET estimations, the former was measured directly from the site and the latter was, to a certain degree, related to the observed EDVI. The solid dots represent the clear-sky cases when NDVI values were retrieved. Since changes in solar radiation from day to day under clear-sky condition were small, variations in ET fluxes were the consequence of vegetation changes quantified by the EDVI values. The statistical results from clear-sky conditions are comparable to those from other leading remote sensing techniques, such as from NDVI under clear-sky conditions [Nishida et al., 2003]. Thus, generally, EDVI has comparable or better relation to the fluxes of atmosphere-ecosystem exchange than NDVI. Since EDVI captures the seasonal changes of leaf physiology and is directly related to key factors that determines photosynthesis processes, particularly vegetation moisture content and radiation use efficiency, and carbon uptakes can be simply described as a product of PAR and carbon efficiency [Min 2005], we use the product of EDVI and PAR to predict the carbon uptake. Similar relationship $(\mathrm{R}=0.74)$ between $\mathrm{CO}_{2}$ uptake fluxes and the products of the EDVI and surface PAR measurements at Harvard Forest is shown in Fig. 7b. As pointed out by Min [2005], radiation-use-efficiency varies significantly from clear-sky, to partial cloudy, and to overcast conditions. A simple linear relationship in all data shown in Figure $7 \mathrm{~b}$ cannot high-accurately explain changes of $\mathrm{CO}_{2}$ fluxes since these data contain measurements under both clear-sky and cloudy conditions. The correlation coefficient of EDVI with $\mathrm{CO}_{2}$ fluxes for clear-sky cases when NDVI measurements were available, 
solid dots in the Figure $7 \mathrm{~b}$, is 0.97 , much higher than simple overall regression.

Observational uncertainties in the current analysis are considerable. Certain assumptions also introduce errors in ET and $\mathrm{CO}_{2}$ flux estimations, such as spatial and temporal inconsistencies between the satellite measurements and the surface observations. The footprint of tower flux measurements is only a small fraction of footprints of SSM/I sensors. Scale-inconsistencies have certain effects on the evaluation and comparison of the satellite and surface data. Uncertainties associated with ground observations of ET and $\mathrm{CO}_{2}$ uptake fluxes are the other example. These fluxes were not directly measured at the site. Instead, they were calculated from covariance methods. Small errors in meteorological variable measurements may produce non-negligible errors in these fluxes. In general, although some variances shown in this study may be explained by scale-inconsistence and observational uncertainties, majority variations of the ET and $\mathrm{CO}_{2}$ fluxes are reflected in the remotely sensed EDVI data, as shown in our comparisons.

\section{Conclusions}

In this study, we introduced a new index, EDVI, based on the emissivity difference between two wavelengths to understand canopy VWC and associated forest-atmosphere exchanges. A simple conceptual two-layer model for microwave radiative transfer calculations is used to illustrate the fundamental physical linkage of EDVI to crown canopy VWC in heavy forest environments. It shows that EDVI is insensitive to the soil moisture and has a nearlinear relation to the VWC for the range of VWC in some canopies. We further used a combination of visible, infrared and microwave measurements to retrieve EDVI from satellite SSM/I measurements and to estimate forest-atmosphere exchanges. The satellite estimated EDVI captures the seasonal changes of leaf physiology, the overriding feature of deciduous forest canopy. This study demonstrates the potential of the combined microwave, visible and infrared remote sensing technique in monitoring vegetation biomass and the consequence of ecosystem exchange processes, particularly under cloudy conditions where cloud-vegetation interaction takes place, and NDVI technique is not available. The EDVI is found empirically sensitive to EF under all-sky conditions. Under clear-sky conditions when NDVI values can be retrieved, EDVIv estimates show stronger relationships to $\mathrm{EF}$ and $\mathrm{CO}_{2}$ uptake than NDVI retrievals. This, along with the characteristics of microwave land 
surface emissivity, suggests that the EDVI value could be used to represent the physiological characteristics and responses of the canopy. Furthermore, the simple linear products of observed EDVI and input energy (SW and PAR) values are significantly correlated to $\mathrm{ET}(R=0.95)$ and $\mathrm{CO}_{2}$ uptake $(R=0.74)$. These empirical relationships between the microwave indices and $\mathrm{ET}$ and $\mathrm{CO}_{2}$ uptake illustrate a potential for combined satellite retrievals of

microwave, infrared and visible measurements to estimate land surface-atmosphere exchanges. With a more complex forest-atmosphere exchange model with proper aerodynamic processes, the estimation of exchange fluxes using EDVI will be significantly improved. More importantly, EDVI values are less sensitive to aerosols and clouds than those from visible and near-infrared measurements, and can be obtained during both day and night times under all-weather conditions except precipitation. Although the spatial resolution of microwave measurements is generally lower than visible and near-infrared observations, effective temporal sampling rates of required datasets will be increased. Further investigations on the physical relationships between EDVI values and biophysical parameters of canopies and on other canopy types are needed. Particularly, a better model, which includes meteorological conditions, is needed to link remotely sensed biophysical parameters to the exchange processes of land surface and atmosphere.

\section{Acknowledgements:}

This research was supported by the Office of Science (BER), U.S. Department of Energy, through the Atmospheric Radiation Measurements (ARM) and Northeast Regional Center (NRC) of the National Institute for Global Environmental Change (NIGEC) under Cooperative Agreement No. DE-FC03-90ER61010. The support is also from the Earth System Science Research Program under NASA Earth Observing System (EOS) Mission and the NASA Energy and Water cycle Study (NEWS) and EOS science and data analysis programs. SSM/I data were downloaded from NASA MSFC data center, NDVI data were downloaded from NASA GSFC DAAC, and surface data were obtained from the NIGEC-NRC at Harvard University.

\section{References:}

Asrar, G. E., M. Fuchs, E. T. Kanemasu, J. L. Hatfield, Estimating absorbed photosynthetic radiation and leaf area index from spectral reflectance in wheat, Agron. J. 76, 300-306, 1984. 
Choudhury, B. J., Synergism of optical and microwave observations for land surface studie, Passive microwave remote sensing of land-atmosphere interactions, edited by B. J. Choudhury, Y. H. Kerr, E. G. Njoku and P. Pampaloni, Netherlands, 1995.

Crago, R. D., Comparison of the evaporative fraction and the Priestley-Taylor $\alpha$ for parameterizing daytime evaporation, Water Resour. Res., 32(5), 1403-1409, 1996.

Ferrazzoli, P. and L. Guerriero, Passive microwave remote sensing of forests: a model investigation IEEE Trans. Geosci. Remote Sens. 34, 433-43, 1996.

Granger, R. J., Satellite-derived estimates of evaportranspiration in the Gediz basin, J. of Hydro. 229, 70-76, 2000.

Hansen, J. E., and L. D., Travis, Light scattering in planetary atmosphere, Space Sci. Rev. 16, 527-610, 1974.

Jiang, L., and S. Islam, An intercomparison of regional latent heat flux estimation using remote sensing data, Int, J. Remote Sensing, 24, 2221-2236, 2003.

Jackson, T. J., \& Schmugge, T. J., Vegetation effects on the microwave emission of soils. Remote Sensing of Environment, 36, 203-212, 1991.

Jackson, T. J., \& Schmugge, T. J., and P. O'Neill, Passive microwave remote sensing of soil moisture from an Aircraft platform, Remote sensing of environment, 14, 135-151, 1984.

Jackson, T. J., D. Chen, M. Cosha, F. Lia, M. Andersonc, C. Walthalla, P. Doriaswamya, E. R. Hunta, Vegetation water content mapping using Landsat data derived normalized difference water index for corn and soybeans, Remote Sensing of Environment 92, 475-482, 2004.

Lin, B., B. Wielicki, P. Minnis, and W. B. Rossow, Estimation of water cloud properties from satellite microwave, infrared, and visible measurements in oceanic environments. I: Microwave brightness temperature simulations, J. Geophys. Res., 103, 3873-3886, 1998.

Lin, B., and P. Minnis, Temporal variations of land surface microwave Emissivities over the atmospheric radiation measurement program southern great plains site, J. Apple. Meteor., 39, 1103-1116, 2000.

Lin, B., P. Minnis, A. Fan, J. Curry, and H. Gerber, Comparison of cloud liquid water paths derived from in situ and microwave radiometer data taken during the SHEBA/FIREACE, Geophys. Res. Letters, 28, 975-978, 2001.

Macelloni, G., S. Paloscia, P. Pampaloni and E. Santi, Global scale monitoring of soil and vegetation using SSM/I and ERS wind scatterometer, J. Int. J. Rem. Sens., 24, 2409-2425, 2003. 
McClathey, R.A., R.W. Fenn, J.E.A. Selby, F.E. Voltz, and J.S. Garing, 1972: Optical properties of the atmosphere. Air Force Cambridge Research Laboratories, AFCRL-72-0497, Environ. Res. Pap., No. 411.

Min, Q.-L, Impacts of aerosols and clouds on CO2 uptake over Harvard Forest, J. Geophys. Res, Vol. 110, No. D6, D06203, doi:10.1029/2004JD004858, 2005.

Moore, K. E., D. R. Fitzjarald, R.K. Sakai, M.L. Goulden,J.W. Munger, and S. C. Wofsy, Seasonal variation in radiative and turbulent exchange at a deciduous forest in Central Massachusetts, J. Appl. Met., 35, 122-134, 1996.

Myneni, R. B., F. B. Hall, P. J. Sellers, A. L. Marshak, The interpretation of spectral vegetation indices, IEEE Trans. GeoSci. Remote Sens. 33:481-486, 1995.

Neale, C. M. U., M. J. McFarland, and K. Chang, Land-Surface-Type Classification Using Microwave Microwave/Imager Brightness Temperatures From the Special Sensor, IEEE Trans. GeoSci. Remote Sens. 28, 828-839, 1990.

Nishida, K., R. R. Nemani, S. W. Running, and J. M. Glassy, An operational remote sensing algorithm of land surface evaporation, J. Geophy. Res., 108(D9), 427, doi:10.1029/2002JD002062, 2003.

Njoku E. G., AMSR Land Surface Parameters: Surface Soil Moisture, Land Surface Temperature, Vegetation Water Content, ATBD of AMSR-E Science Team, NASA EOS Project, 1999.

O’keef, J., Woody species phenology, Prospect Hill Tract, Harvard Forest-2003, in Proceedings of Harvard University LTER and NIGEC programs, Harvard Forest, 2004.

Paloscia, S., Microwave emission from vegetation, Passive microwave remote sensing of land-atmosphere interactions, edited by B. J. Choudhury, Y. H. Kerr, E. G. Njoku and P. Pampaloni, Netherlands, 1995.

Pinker, R. T., et al., Surface radiation budgets in support of the GEWEX Continental-Scale International Project (GCIP) and the GEWEX Americas Prediction Project (GAPP), including the North American Land Data Assimilation System (NLDAS) project, J. Geophys. Res., 108(D22), 8844, doi:10.1029/2002JD003301, 2000.

Robinson, D.A., K.F. Dewey and R.R. Heim, Global snow cover monitoring: An update. Bulletin of American Meteorological Society, 74, 1689-1696, 1993.

Pulliainen, J. T., J. Grandell, and M. T. Hallikainen, HUT Snow Emission Model and its Applicability to Snow Water Equivalent Retrieval, IEEE Trans. GeoSci. Remote Sens. 37, 1999.

Sellers, P. J., Canopy reflectance, photosynthesis, and transpiration, Intl. J. Remote Sens. 6, 1335-1372, 1985. 
Shuttleworth, W. J., R. J. Gurney, A. Y. Hsu, and J. P. Ormsby, FIFE: The variation in energy partition at surface flux sites, IAHS Publ., 186, 67-74, 1989.

Sims D., and J. A. Gamon1, Estimation of vegetation water content and photosynthetic tissue area from spectral reflectance: a comparison of indices based on liquid water and chlorophyll absorption features, Remote Sensing of Environment, 84 526-537, 2003.

Ulaby, F. T., R. K. Moore, and A. K. Fung, Microwave Remote Sensing-Active and Passive., vol. III, From Theory to Applications, London, U.K.: Artech House, 1986.

Van de Griend, A. A., and J.-P.Wigneron, On the Measurement of Microwave Vegetation Properties: Some Guidelines for a Protocol, IEEE Trans. Geosci. Remote Sensing, 42, 2277-2289, 2004.

Waring, R. H., and S. W. Running, Forest Ecosystems: analysis at multiple scales, second edition, Academic Press, 1998.

Wigneron, J.-P., J.-C. Calvet,, Y.H. Kerr, A. Chanzy, and A. Lopes, Microwave emission of vegetation: sensitivity to leaf characteristics, IEEE Trans. Geosci. Remote Sensing, 31, 716-726, 1993.

Wigneron, J.-P., J.-C. Calvet, T. Pellarin, A.A. Van de Griend, M. Berger, P. Ferrazzoli, Retrieving near-surface soil moisture from microwave radiometric observations: current status and future plans, Remote Sensing of Environment, 85, 489-506, 2003.

Wofsy, S. C., M. L. Goulden, J. W. Munger, S.-M. Fan, P. S. Bakwin, B. C. Daube, S. L. Bassow, and F. A. Bazzaz, Net exchange of $\mathrm{CO}_{2}$ in midlatitude forests, Science, 260, 2224-2238, 1993.

Zarco-Tejada, P.J., C.A. Rueda, S.L. Ustin, Water content estimation in vegetation with MODIS reflectance data and model inversion methods, Remote Sensing of Environment, 85 109-124, 2003. 


\section{Crown Layer: Leaves, Needles, and Twigs}

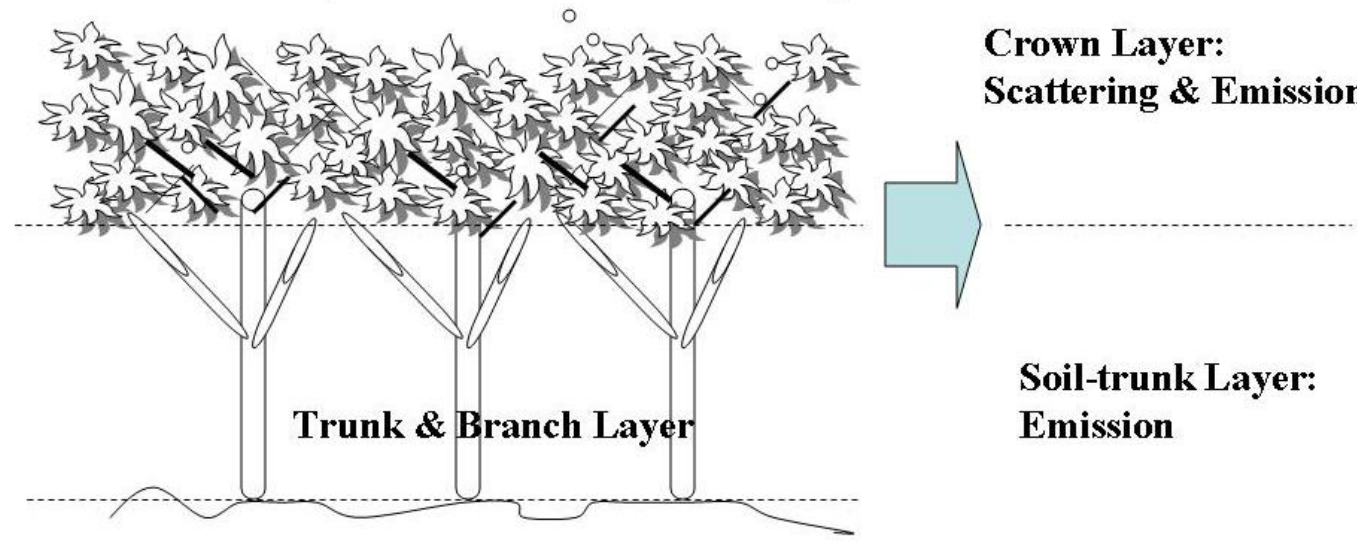

Soil Layer

Figure 1. Sketch of soil-forest medium for microwave radiative transfer models. The conceptual soil-trunk layer on right side is used for simplifications in model simulations.
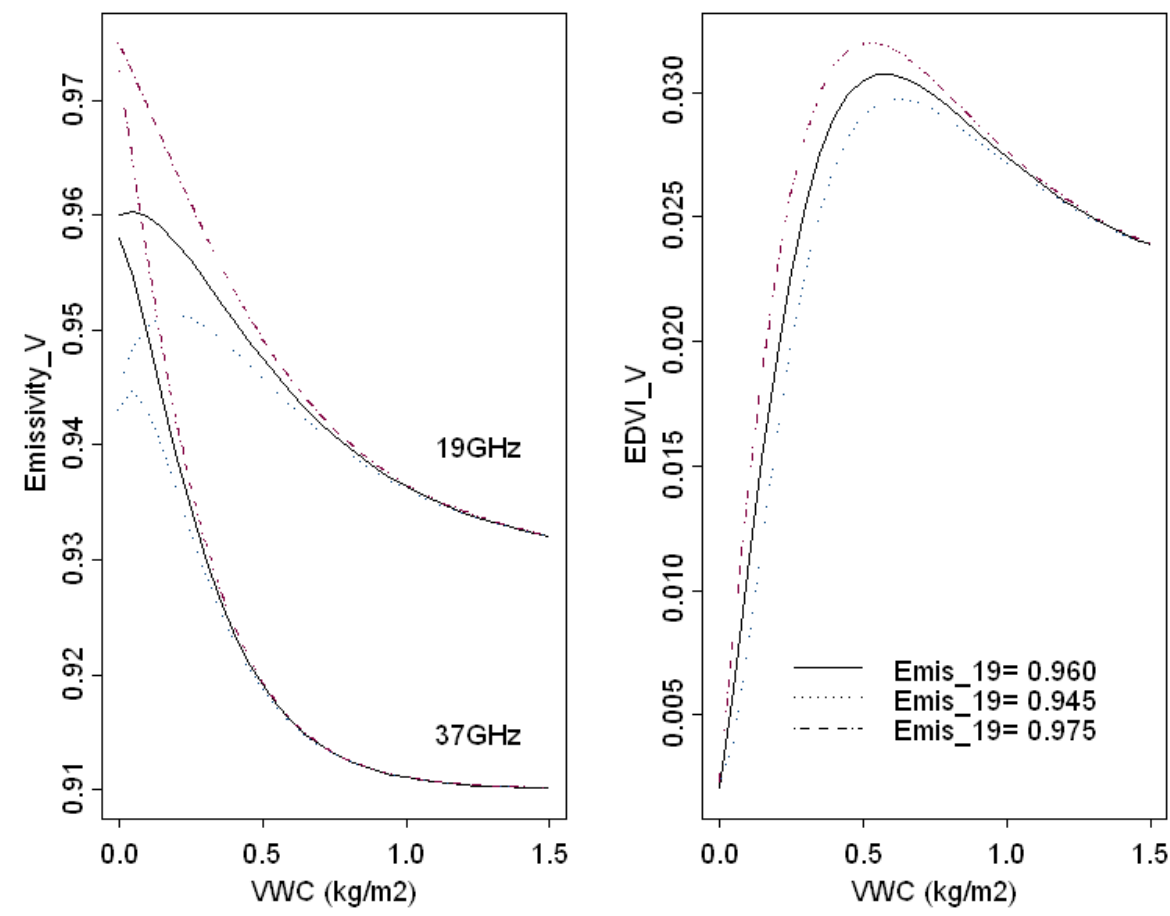

Figure 2. Simulated canopy emissivities at 19.4 and $37 \mathrm{GHz}$ and $\mathrm{EDVI}_{\mathrm{V}}$ as a function of VWC of canopy crown layer for different soil-trunk layer emissivities. Solid lines represent the basic condition (Emis_19=0.960 and Emis_37=0.958) of soil-trunk layer. Dotted lines and dashed lines represent wetter or drier conditions, respectively. 


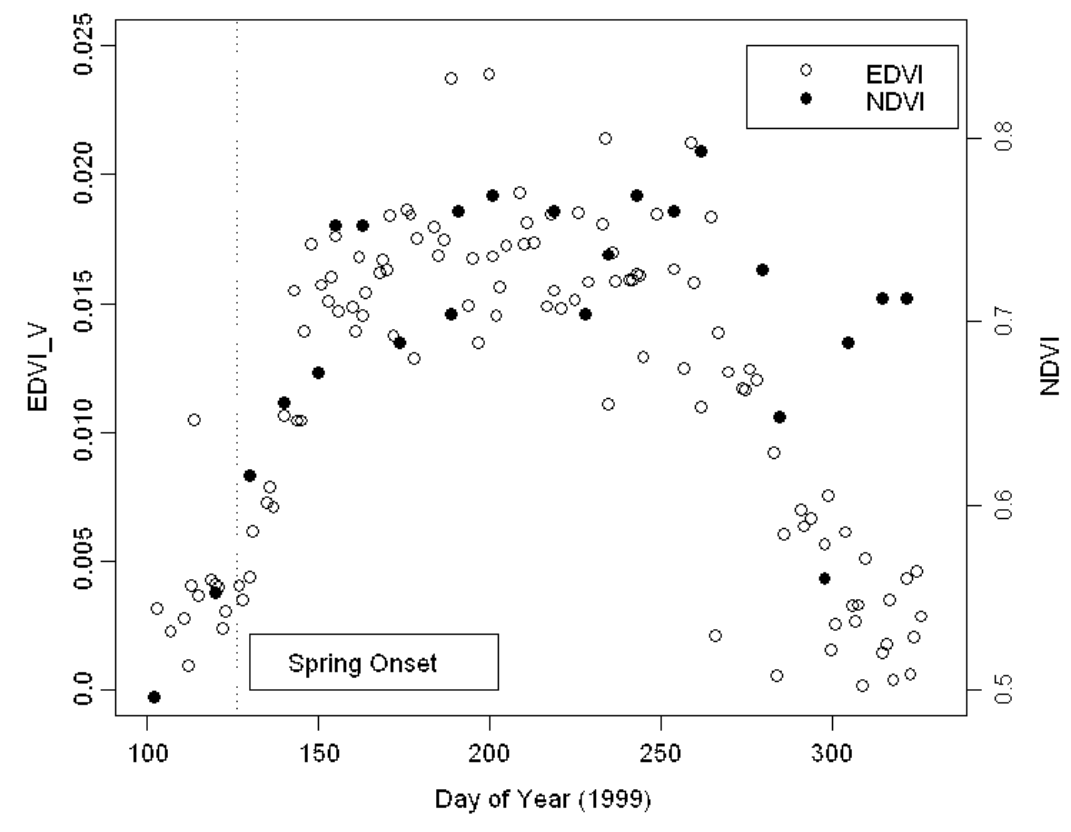

Figure 3. Retrieved vertically polarized EDVI $\mathrm{V}$ and NDVI at the Harvard Forest site during the growing season of 1999.
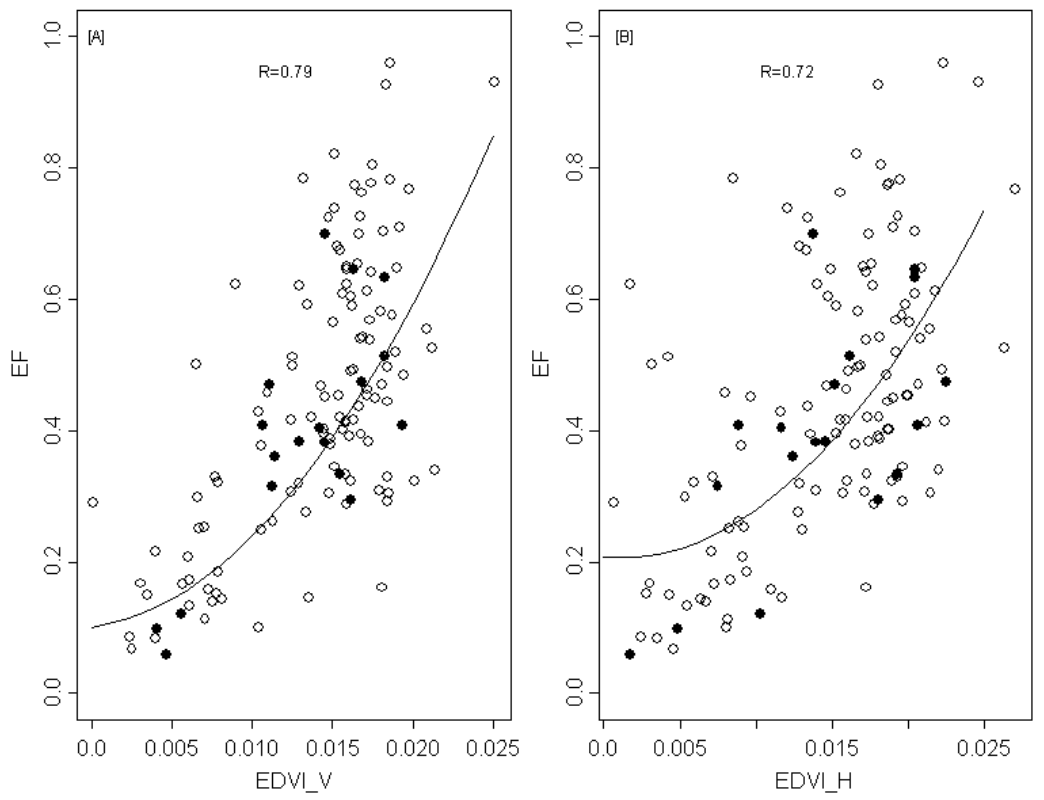

Figure 4. Scatterplots of the derived EF at the Harvard Forest site against the satellite retrieved EDVI values for both vertical and horizontal components during growing seasons of 1999 (DOY 128-315) and 2000 (DOY 130320). The solid dots represent cases under clear-sky conditions when NDVI values can be retrieved. The lines represent a least squared fitting. 

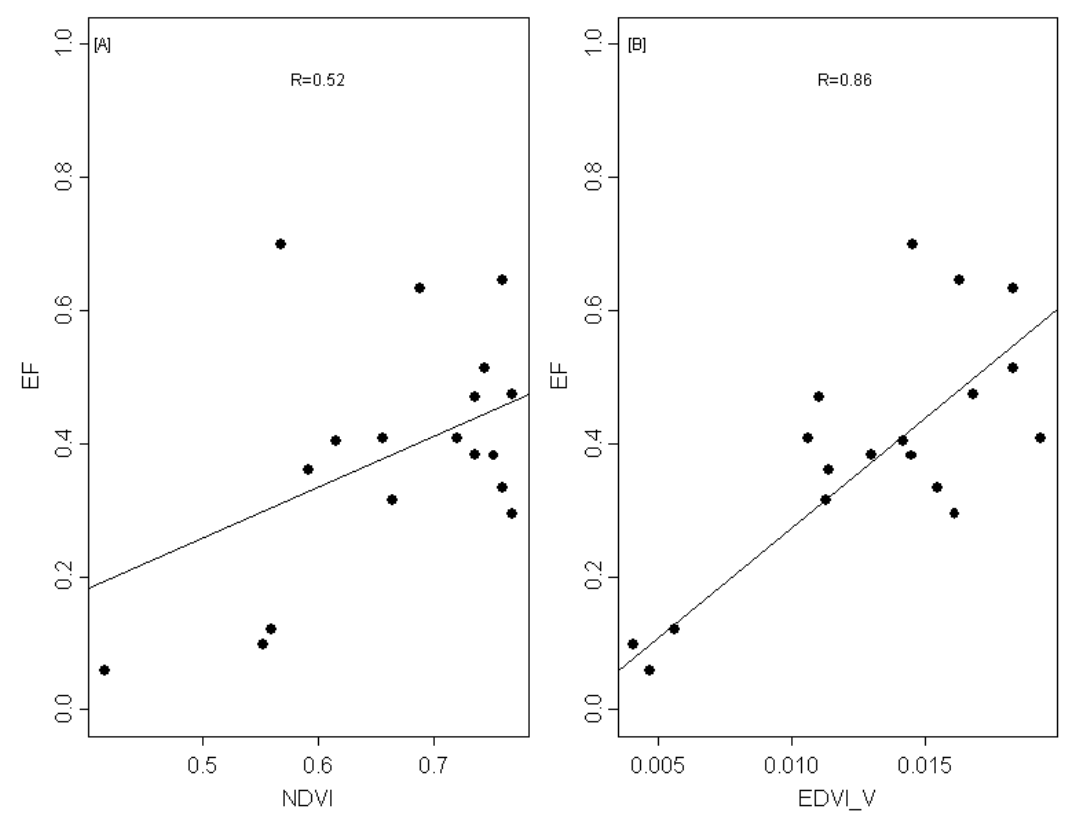

Figure 5. Scatterplots of the derived EF at the Harvard Forest site against the satellite retrieved EDVI and NDVI values for growing seasons of 1999 and 2000 when NDVI values can be retrieved. The lines represent a least squared fitting.
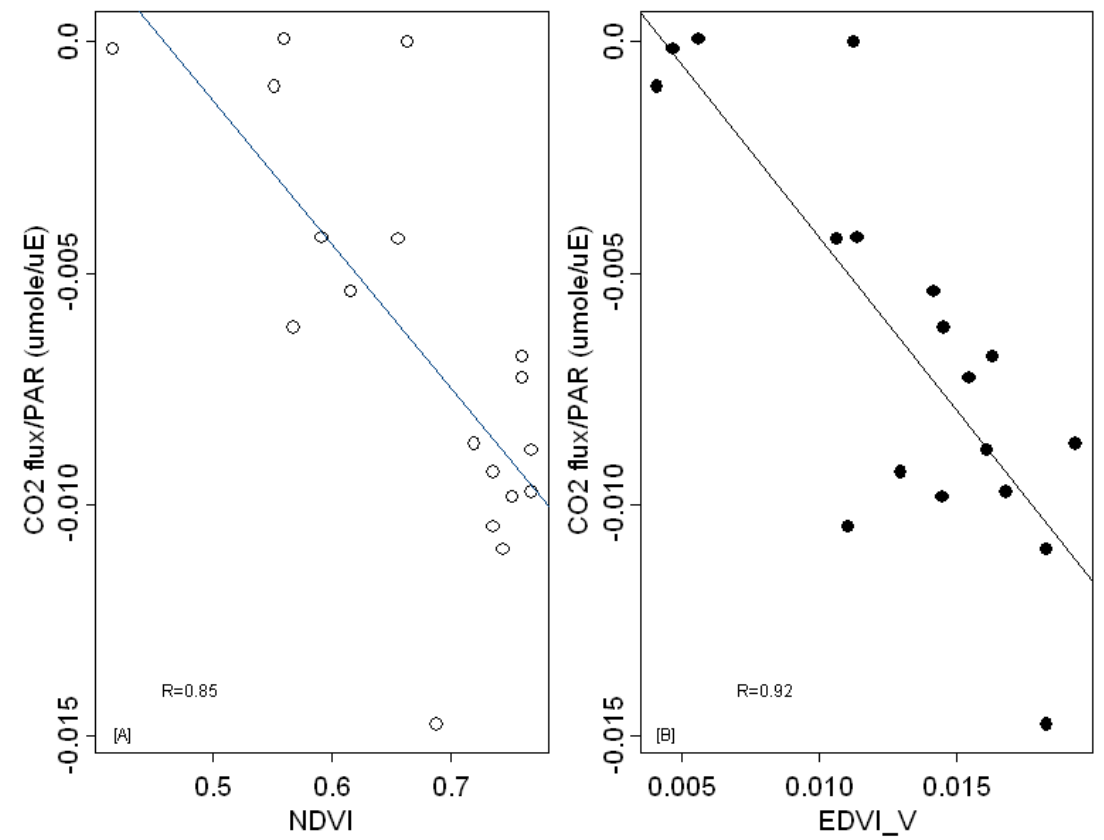

Figure 6. Observed ratio of $\mathrm{CO}_{2}$ uptake flux to PAR as a function of NDVI and EDVIv. The solid line represents a linear least squared fitting. 

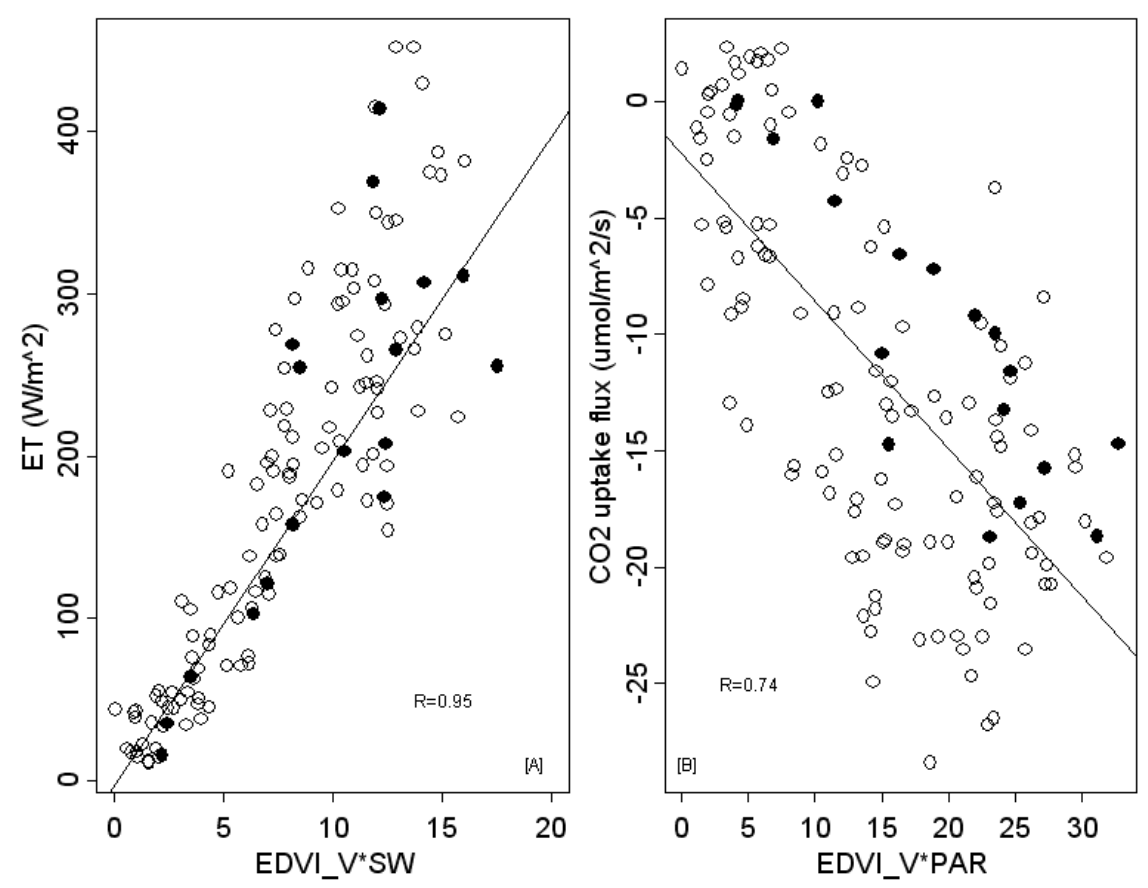

Figure 7. Observed ET as a function of $\mathrm{EDVI}_{\mathrm{V}}{ }^{*} \mathrm{SW}$ and measured $\mathrm{CO}_{2}$ uptake fluxes as a function of $\mathrm{EDVI}_{\mathrm{V}} * \mathrm{PAR}$, respectively. 